\title{
ÜBER DIE KLASSENZAHLEN ALGEBRAISCHER ZAHLKÖRPER
}

\author{
SIGEKATU KURODA
}

Seit Herr Artin seine allgemeinen L-Funktionen, die mit Frobeniusschen Gruppencharakteren gebildet sind, entdeckt hat [1], sind die multiplikativen Relationen Dedekindscher $\zeta$-Funktionen als additive Relationen zwischen den Frobeniusschen Gruppencharakteren mit Erfolg untersucht worden. Durch diese Methode sind gewisse Klassenzahlrelationen in den folgenden Zeilen zu betrachten.

Eine additive Relation (1) zwischen den Gruppencharakteren bringt nämlich die entsprechende multiplikative sowohl zwischen den $\zeta$-Funktionen (2), als auch, wegen Artinscher Führerdiskriminantenformel, zwischen den Diskriminanten relativ-galoisscher Körper (6) hervor. Daraus ergibt sich eine Klassenzahlrelation (12), die darauf hinweisen möchte, dass zwischen Idealkassen- und Einheitengruppe gewisser innige Zusammenhang steckte. Etwaige Formel (12) lässt sich im relativ-abelschen Falle mit Hilfe der Möbiusschen Funktion verwirklichen (15).

Indem dies auf den speziellen abelschen Körper vom Grade $l^{m}$, dessen Galoisgruppe vom Typus $(l, l, \ldots, l)$ ist, angewandt wird, erhalten wir die Formel (17; Dass die Zahl $\mathbf{A}$ in (17) eine Potenz von $l$ ist, folgt algebraisch aus dem va: Nehrkorn [5] herrührenden Satze (19). Unter der Annahme, die zu Anfang de Nr. 7 gestellt wird, werden wir in (38) den expliziten Ausdruck von A geber, der eine teilweise Verallgemeinerung des Dirichlet-Herglotz'schen Satzes [4] ist.

\section{LITERATURVERZEICHNIS}

[1] E. Artin: Über neue Art von L-Reihen, Abh. Math. Sem. Hamburg, 3 (1924).

[2] — : Die Gruppentheoretische Struktur der Diskriminanten algebraischer Zahlkörper, Journ. für reine und angew. Math., 164 (1931).

[3] — : Zur Theorie der L-Reihen mit allgemeineren Gruppencharakteren, Abh. Math. Sem. Hamburg, 8 (1931).

[4] G. Herglotz, Über einen Dirichlet'schen Satz, Math. Zeitschr. 12 (1922).

[5] H. Nehrkorn, Über absolute Idealklassengruppen und Einheiten in algebraischen Zahlkörpern, Abh. Math. Sem. Hamburg, 9 (1939).

Received March 9, 1950. 
1. Es sei $K / k$ ein relativ-galoisscher Zahlkörper mit der Galoisgruppe $G$ und $\chi_{\Omega}(\sigma)(\sigma \in G)$ der Charakter von $G$, der durch den Hauptcharakter der dem Uuterkörper $\Omega$ von $K$ entaprechenden Untergruppe $H$ von $G$ induziert.

Erstens ist dann nach Artin [3]

$$
\zeta_{\Omega}(s)=L\left(s, \chi_{\Omega} ; K / k\right),
$$

wobei $\zeta_{\Omega}(s)$ die zu $\Omega$ gehörige Dedekindsche $\zeta$-Funktion und $L\left(s, \chi_{\Omega} ; K / k\right)$ die zu $\chi_{\Omega}$ gehörige Artinsche $L$-Funktion ist. Wenn $z$ wischen $\chi_{\Omega \Omega}(\sigma)$ eine lineare Relation

$$
\sum_{\Omega} c_{\Omega} \chi_{\Omega}(\sigma)=0
$$

für alle $\sigma \in G$ mit ganzrationalem $c_{\Omega}$ besteht, so erhalten wir dementsprechend eine multiplikative Relation

$$
\Pi \zeta_{\Omega}(s)^{c_{\Omega}}=1
$$

Nach (1) ist speziell für $\sigma=1$

$$
\sum c_{\Omega} m_{\Omega}=0,
$$

wobei $m_{\Omega}=\%_{\Omega}(1)$ die Ordnung der Faktorgruppe $G / H$ oder der Körpergrad $(\Omega / k)$ sind.

Zweitens haben wir auch nach Artin [2] die Führerdiskriminantenformel

$$
D(\Omega / k)=\mathfrak{f}(\%, K / k),
$$

wobei $D(\Omega / k)$ die Relativdiskriminante von $\Omega / k$ und $\mathrm{f}\left(\chi_{\Omega}, K / k\right)$ der Führer des Charakters $\psi_{\Omega}$ bezüglich $K / k$ ist. Unter der Voraussetzung (1) folgt aus (4) unmittelbar die Formel

$$
\Pi D(\Omega / k)^{\varsigma_{\Omega}}=1 \text {. }
$$

Durch absolute Normbildung der Differente $\mathfrak{D}(\Omega)=\mathfrak{D}(\Omega / k) \mathfrak{D}(k)$ unter Benutzung von (3) führt die Formel (5) weiter auf die zwischen den Diskriminanten $D(\Omega)$ der Unterkörper $\Omega$ von $K / k$ bestehende Formel

$$
\left|\Pi D(\Omega)^{c_{\Omega}}\right|=1 \text {. }
$$

Drittens definieren wir für einen Charakter $\chi$ der Gruppe $G$ und für eine Bewertung $\mathfrak{p}$ von $k$ die folgende Zahl

$$
\beta(\%, \mathfrak{p}, K / k)= \begin{cases}(2 \pi)^{x(1)}, & \text { wenn } \mathfrak{p} \text { komplex ist, } \\ 2^{\left[x_{(1)}+x_{(\sigma)}\right] / 2} \pi^{[x(1)-x(\sigma)] / 2}, & \text { wenn } \mathfrak{p} \text { reell ist. }\end{cases}
$$

Dabei bezeichnen wir mit $\sigma$ die Frobenius-substitution einer Primteiler $\mathfrak{P}$ von $\mathfrak{p}$ in $K$. Wenn weiter

$$
B(\chi, K / k)=\prod_{\mathfrak{p}} \beta(\chi, \mathfrak{p}, K / k),
$$

gesetzt wird, wobei $\mathfrak{p}$ alle unendlichen Primstelle von $k$ durchlaufe, so können wir beweisen wie bei Artinschen Gammafaktoren [3]

$$
\begin{aligned}
& B\left(\chi_{1}+\chi_{2}, K / k\right)=B\left(\chi_{1}, K / k\right) B\left(\chi_{2}, K / k\right), \\
& B\left(\chi_{,} K^{\prime} / k\right)=B(\chi, K / k),\left(K^{\prime} \supseteqq K, K^{\prime} / k \text { galoissch }\right), \\
& B\left(\chi_{\psi}, K / k\right)=B(\psi, K / \Omega),(\psi \text { ein Charakter von } H) .
\end{aligned}
$$


Für den vom Hauptcharakter von $H$ induzierten Charakter $\%$ gilt nun wegen (7) und (8)

$$
B(\%, K / k)=2^{r_{1}{ }^{\Omega}+r_{2}{ }^{2}} \pi^{r_{2} 2^{2}},
$$

wobei $r_{1}^{\Omega}, \boldsymbol{r}_{2}^{\Omega}$ bezüglich der Anzahl der konjugierten Körper von $\Omega$ die übliche Bedeutung haben. Wegen (8) und (9) erhalten wir dann aus unserer Voraussetzung (1)

$$
\prod_{\Omega}\left(2^{r_{1}{ }^{2}+r_{2}{ }^{\Omega}} \pi^{r_{\Omega}{ }^{\Omega}}\right)^{c_{\Omega}}=B\left(\sum c_{\Omega} \%, K / k\right)=1 .
$$

Speziell gilt $\left(r_{\Omega}=r_{1}^{\Omega}+r_{2}^{\Omega}-1\right)$

$$
\sum c_{\Omega} r_{1}^{\Omega}=\sum c_{\Omega} r_{2}^{\Omega}=\sum c_{\Omega} r_{\Omega}=\sum c_{\Omega}=0 \text {. }
$$

Wenn letztens mit $h_{\Omega}, R_{\Omega}, w_{\Omega}$ die Klassenzahl, der Regulator bzw. die Anzahl der Einheitswurzeln des Körpers $\Omega$ bezeichnet werde, so ergibt sich nach (2)

$$
\prod_{\Omega}\left(\lim _{s \rightarrow 1}(s-1) \zeta_{\Omega}(s)\right)^{c_{\Omega}}=\prod_{\Omega}\left(h_{\Omega} \frac{2^{r_{1}{ }^{\Omega}+r_{2}{ }^{2}} \pi^{r_{2}{ }^{2}} R_{\Omega}}{w_{\Omega} \sqrt{\mid \overline{D(\Omega) \mid}}}\right)^{c_{\Omega}}=1 \text {. }
$$

Daraus folgt wegen (6) und (10) die Klassenzahlrelation

$$
\Pi h_{\Omega} c_{\Omega}=\Pi\left(\frac{w_{\Omega}}{R_{\Omega}}\right)^{c_{\Omega}} \text {. }
$$

2. Um wirklich eine Formel (1) für relativ-abelschen Körper zu finden, schalten wir eine Betrachtung über beliebige Gruppe $G$ ein. Sei $N$ ein Normalteiler von $G$, so ist ersichtlich der durch den Hauptcharakter von $N$ induzierte Charakter $\psi_{N}(\sigma)=(G: N)$ oder 0 , je nachdem $\sigma \in N$ oder $\sigma \notin N$ ist. Mithin gilt

$$
\chi_{v v}(\sigma)=\sum_{j} f_{j} \chi_{j}(\sigma)=\sum_{i} f_{i} \Xi_{i}(\sigma), f_{j}=\chi_{j}(1) .
$$

wobei die erste Summe alle einfachen Charaktere $\chi_{j}$ von $G / N$, die letzte alle rationalen Charaktere $\Xi_{i}$, die die Summe $\Xi_{i}(\sigma)=\sum \chi(\sigma)$ algebraisch konjugierter einfacher Charaktere $\%(\sigma)$ von $G / N$ sind, durchlaufe.

Nun sei $G$ abelsch und werde mit $U, V, W, \ldots$ beliebige Intergruppe von $G$ mit zyklischer Faktorgruppe $G / U, \ldots$ bezeichnet. Dann entspricht jeder $U$ eineindeutig ein rationaler Charakter $\Xi_{l^{-}}$in der Weise, dass $\Xi_{l}(\sigma)=\Xi_{C^{-}}(1)$ dann und nur dann gilt, wenn $\sigma \in U$ ist. Damit ergibt sich nach (13) für jede Untergruppe $N$ von $G$

$$
\psi_{x}(\sigma)=\sum_{l: Y} \Xi_{L^{\prime}}(\sigma)
$$

wobei die Summe alle Charaktere $\Xi_{C}$, die der Gruppe $U \supset N$ mit zyklischer $G / U$ entsprechen, durchlaufe. Mit Hilfe der Möbiusschen Funktion $\mu$ folgt nun aus (14) für eine feste $W$

$$
\sum_{i \supset W} \mu(U: W) \%_{U}(\sigma)=\sum_{U>W} \mu(U: W) \sum_{i>U} \Xi_{V}(\sigma)=\sum_{i=W} \Xi_{V} \cdot(\sigma) \sum_{r=U \supset W} \mu(U: W)
$$

Weil aber $V / W$ zyklisch ist, ist also

$$
\sum_{V=U D W} \mu(U: W)=\sum_{d /(V: W)} \mu(d)=\left\{\begin{array}{lll}
1 & \text { für } \begin{array}{l}
V=W \\
\\
0
\end{array} & V \neq W .
\end{array}\right.
$$


Daher erhält man

$$
\Xi_{W}(\sigma)=\sum_{c} \mu(U: W) \chi_{V^{*}}(\sigma) .
$$

Daraus folgt wegen (14) für die Einheitsgruppe $E$

$$
\chi_{E}(\sigma)=\sum_{W} \Xi_{W}(\sigma)=\sum_{W} \sum_{U \supset W} \mu(U: W) \chi_{U}(\sigma)=\sum_{\tau} \chi_{v}(\sigma) \sum_{t \supset W} \mu(U: W) .
$$

Wenn also

$$
c_{C^{r}}=\sum_{U \supset W} \mu(U: W)
$$

gesetzt wird, erhalten wir die Formel

$$
\gamma_{E}(\sigma)=\sum_{U} c_{U} \chi_{U}(\sigma)
$$

Diese Formel gibt nach (1) und (12) eine Klassenzahlrelation zwischen den Unterkörpern des relativ-abelschen Körper $K / k$ mit der Galoisgruppe $G$.

Spezialisieren wir die Formel (15) für einen relativ-abelschen Körper $K / k$ vom Primzahlpotenzgrade $l^{m}$, dessen Galoisgruppe vom Typus $(l, l, \ldots, l)$ ist, so ergibt sich

$$
\chi_{\mathrm{K}}-\chi_{k}=\sum_{\Omega}\left(\chi_{\Omega}-\chi_{k}\right),
$$

wobei die Summe die $t=\left(l^{m}-1\right) /(l-1)$ zyklischen Unterkörper $\Omega / k$ vom Relativgrade $l$ durchlaufe. Aus (12) und (16) folgt

$$
\begin{gathered}
\frac{H}{h}=\mathbf{A} \prod_{j=1}^{t} \frac{h_{j}}{h}, \\
\mathbf{A}=\mathbf{P} \cdot \mathbf{W}, \quad \mathbf{P}=\frac{R(k)}{R(K)} \prod_{j=1}^{t} \frac{R\left(\Omega_{j}\right)}{R(k)}, \quad \mathbf{W}=\frac{W}{w} \prod_{j=1}^{t} \frac{w}{w_{j}},
\end{gathered}
$$

wobei $(H, R(K), W),(h, R(k), w)$ bzw. $\left(h_{j}, R\left(\Omega_{j}\right), w_{j}\right)$ dieselbe Bedeutung wie oben bezüglich des Körpers $K, k$ bzw. der $t$ Unterkörper $\Omega_{j}$ hat.

3. Wir wollen die Zahl A später näher bestimmen. Allerdings können wir ohne Benutzung der $\zeta$-Funktion beweisen, dass $\mathbf{A}$ eine Potenz von $l$ ist. Nämlich sei $K / k$ beliebiger algebraischer Zahlkörper vom Relativgrade $n$ und vorlaüfig unter Idealklassengruppe verstehen wir immer die gröbere, nämlich die, deren Hauptklasse diejenige Ideale umfasst, welche durch Potenzierung mit dem aus lauter der Primteilern von $n$ komponierten Exponent Hauptideale werden. Dann ist die Untergruppe der Idealklassengruppe $H$ von $K$, deren Klassen ein Ideal von $k$ enthalten, isomorph mit der Idealklassengruppe $h$ von $k$, so dass die Faktorgruppe $H / h$ einen Sinn hat. In unserm abelschen Fall gilt nun der Satz, dass die Faktorgruppe $H / h$ das direkte Produkt der Gruppe $h_{j} / h$ ist:

$$
H / h \cong \prod_{j=1}^{t} h_{j} / h \text {, }
$$

wobei $H, h_{j}$ bzw. $h$ die obengenannte Idealklassengruppe des Körpers $K, \Omega_{j}$ bzw. $k$ bezeichnet [5]. Wir skizieren kurz den Beweis von (19). Dazu braucht es nur die Normbildung der Ideale zu betrachten. 
1) Die Untergruppe von $H / h$, deren Klasse von Idealen aus einem $\Omega_{j}$ erzeugt wird, ist isomorph mit der Gruppe $h_{j} / h$. Denn, wie man leicht aus der Normbildung nach $K / \Omega_{j}$ ersieht, gehört ein Ideal von $\Omega_{j}$ dann und nur dann zur Hauptklasse von $H / h$, wenn das schon $z \mathrm{u}$ derselben von $h_{j} / h$ gehört.

2) Das Produkt $\prod_{j=1}^{t}\left(h_{j} / h\right)$, das nach 1$)$ eine Untergruppe von $H / h$ ist, ist direkt. Denn die Klassen von $H / h$, welche ein Ideal $\Im_{j}$ von $\Omega_{j}$ ( $j$ fest) enthalten und die, welche von einem Produkt $\Im=\prod_{i \neq j} \Im_{i}$ der Ideale $\Im_{i}$ von $\Omega_{i}(i \neq j)$ erzeugt werden, haben keine gemeinsame Klasse ausser Hauptklasse. Dies sieht man wieder durch die Normbildung der Ideale $\Im_{j}$ und $\mathfrak{\Im}$ nach $K / \Omega_{j}$.

3) $\prod_{j=1}^{t}\left(h_{j} / h\right)$ stimmt mit $H / h$ überein. Denn $l^{m-1}$-te Potenz $\Im^{l^{n_{i}-1}}$ aller Ideale $\checkmark$ von $K$ erfüllen alle Klasse der Gruppe $H / h$, da die Ordnung von $H / h$ prim $z$ u $l$ ist. Wenn dann $\Im_{j}=N_{K / \Omega}, \mathfrak{\Im}$ gesetzt wird, das Ideal $\mathfrak{\Im}^{l^{m-1}}$ ist äquivalent in unserm Sinne mit dern Ideale $\prod_{j=1}^{t} \Im_{j}$ aus $\prod_{j=1}^{t}\left(h_{j} / h\right)$, da

$$
\prod_{\jmath=1}^{t} \Im_{j}=\prod_{\jmath=1}^{t} N_{K / \Omega_{j}} \Im=\Im^{t-\left(l^{m-1}-1\right) /(l-1)} N_{K / k} \Im^{\left(l^{m-1}-1\right) /(l-1)},
$$

und dabei $t-\left(l^{m-1}-1\right) /(l-1)=l^{m-1}$ ist. Damit ist (19) bewiesen worden.

Wegen (19) gilt (17) mit der Zahl A, welche eine Potenz von $l$ ist.

4. Um die Zahl A zu bestimmen, brauchen wir die folgenden Bezeichnungen. $\varepsilon, E_{j}, E$ : Einheit des Körpers $k, \Omega_{j}$ bzw. $K$.

$\rho, P_{j}, P$ : Einheitswurzel des Körpers $k, \Omega_{j} \mathrm{bzw} . K$.

$E_{Q}, P_{\Omega}:$ Einheit bzw. Einheitswurzel aus $K$, die durch $E_{j}$ bzw. $P_{j}(j=1, \ldots, t)$ erzeugt wird.

$\boldsymbol{H}_{j} \quad$ : Einheit aus $\Omega_{j}$, für die $N_{\Omega_{j} / k} H_{j}=1$ ist.

$H_{\Omega} \quad$ : Einheit aus $K$, die durch $H_{j}(j=1, \ldots, t)$ erzeugt wird.

$\boldsymbol{r}, \boldsymbol{R}_{\boldsymbol{j}}, \boldsymbol{R}$ : Die Anzahl der Grundeinheiten des Körpers $k, \Omega$, bzw. $K$.

5. Wir betrachten zunächst die Zahl W von (18).

Erstens sei $l \neq 2$. Es sei $p$ eine Primzahl und $W$ genau durch $p^{a}$, dagegen $w$ nicht durch $p^{a}$ teilbar. Dann gibt es ein und nur ein Unterkörper $\Omega_{j}$, die durch Adjunktion der $p^{a}$-ten Einheitswurzel zu $k$ erzeugt wird, weil hierbei $p \neq 2$ sein muss und die Galoisgruppe von $K / k$ vom Typus $(l, l, \ldots, l)$ ist. Daher ist eine und nur eine $w_{j}$ genau durch $p^{a}$ teilbar. Also ist $\mathbf{W}=1$.

Zweitens sei $l=2$. Wenn es in diesem Falle unter den $t$ Körpern $\Omega_{j}$ sowohl $k(\sqrt{-1})$ als auch $k\left(\cos \pi / 2^{a}\right)(a \supseteqq 2)$ gibt, so ist $\mathbf{W}=2$. Sonst ist $\mathbf{W}=1$. Beide Fälle zusammenfassend, erhalten wir

$$
\mathbf{W}=\left(P: P_{\Omega}\right) \text {. }
$$

Nun betrachten wir den Inedx $\left(E_{j}: \varepsilon H_{j}\right)$, der bekanntlich endlich ist. Wenn also $H_{j, 1}, \ldots, H_{j, s_{j}}$ die Basis der Einheitengruppe $H_{j}$ (abgeshen von Einheitswur- 
zeln) sind, so ist $s_{j}=r_{j}-r$ und wegen (11) und (16)

$$
r+\sum_{j=1}^{t} s_{j}=r+\sum_{j=1}^{t}\left(r_{j}-r\right)=R .
$$

Daher besteht das System der Einheiten $H_{j, 1}, \ldots, H_{j, s_{j}}(j=1, \ldots, t)$ und der Grundeinheiten $\varepsilon_{1}, \ldots, \varepsilon_{r}$ von $k$ genau aus $R$ Einheiten von $K$, welche in der Tat unabhägig sind, wie man leicht sieht, wenn man aus der Relation $\varepsilon \prod_{j=1}^{t} H_{j}=1$ die Norm nach $K / \Omega_{j}$ nimmt. Der Regulator der durch diese $R$ Einheiten erzeugten Gruppe werde mit $R\left(\varepsilon H_{\Omega}\right)$ bezeichnet und sei

$$
\begin{aligned}
& \mathbf{P}_{1}=\frac{R\left(\varepsilon H_{\Omega}\right)}{R(K)} \prod_{j=1}^{t} \frac{R\left(\Omega_{j}\right)}{R\left(\varepsilon H_{j}\right)}, \\
& \mathbf{P}_{2}=\frac{R(k)}{R\left(\varepsilon H_{\Omega}\right)} \prod_{j=1}^{t} \frac{R\left(\varepsilon H_{j}\right)}{R(k)}
\end{aligned}
$$

gesetzt. Dann ist $\mathbf{P}=\mathbf{P}_{1} \mathbf{P}_{2}, \mathbf{A}=\mathbf{W P}_{1} \mathbf{P}_{2}$.

6. Die Formel (21) bedeutet $\mathbf{P}_{1}=\frac{\left(E: P_{\varepsilon} H_{\Omega}\right)}{\prod_{j}\left(E_{j}: P_{j} \varepsilon H_{j}\right)}$. Da aber $\left(E: P_{\varepsilon} H_{\Omega}\right)$ $=\left(E: P E_{\Omega}\right)\left(P E_{\Omega}: P \varepsilon H_{\Omega}\right),\left(E: E_{\Omega}\right)=\left(E^{j}: P E_{\Omega}\right)\left(P: P_{\Omega}\right)$ ist, so ist wegen (20)

$$
\mathbf{W P}_{1}=\left(E: E_{\Omega}\right) \frac{\left(P E_{\Omega}: P \varepsilon H_{\Omega}\right)}{\prod_{j}\left(E_{j}: P_{j} \varepsilon H_{j}\right)} .
$$

Nun können wir die Grundeinheiten von $\Omega_{j}$ und die $\varepsilon_{j, 1}, \ldots, \varepsilon_{j, r}$ von $k$ so bestimmen, dass jene entweder aus $\varepsilon_{j, 1}, \ldots, \varepsilon_{j, r}, E_{j, 1}, \ldots, E_{j, s_{j}}$ oder aus $\sqrt[l]{\varepsilon_{j, 1}}, \varepsilon_{j, 2}$, $\ldots, \varepsilon_{j, r}, E_{j, 1}, \ldots, E_{j, s_{j}}$ bestehen. Der letzte Fall entsteht dann und unr dann, wenn $\Omega_{j}=k\left(\sqrt[l]{\varepsilon_{j, 1}}\right)$ ein durch die $l$-te Wurzel einer Einheit von $k$ erzeugte Kummersche Körper ist. Es sei $o_{j}=0$ oder 1 , je nachdem der erste oder der letzte Fall eintritt. Dann ist ersichtlich

$$
\left(E_{j}: P_{j} H_{j}\right)=l^{O},\left(\prod_{\alpha=1}^{s_{j}} E_{j, \alpha}^{x_{j, \alpha}}: \prod_{\alpha=1}^{s_{j}} H_{j, \alpha}^{y_{j, \alpha}}\right) .
$$

Dabei wird die Gruppe der rechten Seite, ebenso wie unten, mod. der durch die ersten $r$ Basen erzeugten Gruppe betrachtet.

Unter den Körpern $\Omega_{j}$, für welche $o_{j}=1$ ist, gebe es genau $u$ unabhängige, etwa $\Omega_{1}=k\left(\sqrt[l]{\varepsilon_{1,1}}\right), \ldots, \Omega_{u}=k\left(\sqrt[l]{\varepsilon_{u, 1}}\right)$. Dann ist $\left(l^{u}-1\right) /(l-1)$ die Anzahl der sämtlichen Körper $\Omega_{j}$ mit $o_{j}=1$. Daher ist

$$
\prod_{j=1}^{t}\left(E_{j}: P_{j} \in H_{j}\left(=l^{\left(l^{u}-1\right) /(l-1)} \prod_{2}\left(\prod_{\alpha} E_{j, \alpha}^{x_{j, \alpha}}: \prod_{\alpha} H_{j, \alpha}^{y_{j, \alpha}}\right)\right. \text {. }\right.
$$

Nun betrachten wir den. Index $\left(P E_{\Omega}: P \varepsilon H_{\Omega}\right)$. Die Gruppe $P E_{\Omega}$ wird, abgesehen von Einheitswurzeln, erzeugt durch die $\sum_{j=1}^{t}\left(r+s_{j}\right)$ Einheiten

$$
\eta_{j}, \varepsilon_{j, 2,}, \ldots, \varepsilon_{j, r}, E_{j, 1}, \ldots, E_{j, s_{j}}, \text { wobei } \eta_{j}=\left\{\begin{array}{l}
\sqrt[1]{\varepsilon_{j, 1}} \\
\varepsilon_{j, 1}
\end{array} \text { für } o_{j}=\left\{\begin{array}{l}
1, \\
0,
\end{array}\right.\right.
$$

von $\Omega_{j}(j=1, \ldots, t)$. Die $r t$ Einheiten $\eta_{j} \varepsilon_{j, 1}, \ldots, \varepsilon_{j, r}(j=1, \ldots, t)$ von dieser 
Reihe erzeugt eine Gruppe mit $r$ Basen, welche mit $\eta$ bezeichnet werde. Weil dann das Element von $E_{\Omega}$ in der Form $\eta \prod_{j=1}^{t} \prod_{\alpha=1}^{s_{j}} E_{j, \alpha}^{x_{j, \alpha}}$ dargestellt wird, deshalb ist der Index $\left(P E_{\Omega}: P \varepsilon H_{\Omega}\right)=\left(\eta \prod_{j} \prod_{\alpha} E_{j, \alpha}^{x_{j, \alpha}}: \varepsilon \prod_{j} \prod_{\alpha} H_{j, \alpha}^{y_{j, \alpha}}\right)=(\eta: \varepsilon)\left(\prod_{j} \prod_{\alpha} E_{j, \alpha}^{x_{j, \alpha}}: \prod_{j} \prod_{\alpha} H_{j, \alpha}^{y_{j, \alpha}}\right)$. Ferner ist ersichtlich, dass aus $\varepsilon_{1,1}^{a_{1}} \varepsilon_{2,1}^{a_{2}} \ldots \varepsilon_{u, 1}^{a_{u}}=\varepsilon^{l}$ notwendigerweise $a_{1} \equiv a_{2} \equiv \ldots$ $\equiv a_{u} \equiv 0$ (mod. $l$ ) folgt, und dass alle $\Omega_{j}$ mit $o_{j}=1$ in der Form

$$
\Omega_{j}=k\left(\sqrt[l]{\varepsilon_{1,1}^{x_{1}} \ldots \varepsilon_{u, 1}^{x_{u}}}\right)
$$

dargestellt werden. Also wegen (25) wird die Gruppe $\eta$ durch die ersten $u$ Systeme $\sqrt[l]{\varepsilon_{j, 1}}, \varepsilon_{j, 2}, \ldots, \varepsilon_{j, r}(j=1, \ldots, u)$ erzeugt. Würde nun $\varepsilon_{j, 1}$ in der Form $\varepsilon_{j, 1}$ $=\varepsilon_{1,1}^{x_{1}} \ldots \varepsilon_{j-1,1}^{x_{j-1}} \varepsilon^{l}$ dargestellt, so widerspricht dies gegen der Unabhängigkeit der Körper $\Omega_{1}, \ldots, \Omega_{u}$. Mithin ergibt sich nach Induktion in bezug auf $j=1, \ldots, u$ $(\eta: \varepsilon)=l^{u}$. Also gilt

$$
\mathbf{W P}_{1}=\left(E: E_{\Omega}\right) l^{u-\left(l^{u}-1\right) /(l-1)} .
$$

7. Nun wollen wir die Zahl $\mathbf{P}_{2}$ betrachten. Von hier ab nehmen wir an, dass jede reelle konjugierte Körper $k_{i}$ von $k$ immer in einem reellen konjugierten Körper $K_{i}$ von $K$ enthalten sei. In diesem Falle sind $R-r=(r+1)\left(l^{m}-1\right)$, $\boldsymbol{r}_{j}-\boldsymbol{r}=(r+1)(l-1)$, so dass alle $s_{j}(j=1, \ldots, t)$ gleich sind, die wir mit $s=(r+1)(l-1)$ bezeichnen wollen. Unsre Annahme ist offenbar erfüllt, falls $l \neq 2$ ist.

Die Substitutionen der Galoisgruppe $G$ von $K / k$ seien $\sigma_{1}=1, \sigma_{2}, \ldots, \sigma_{n}\left(n=l^{m}\right)$ und wir wählen $r+1$ Isomorphismen von $K$, etwa $\theta_{1}, \ldots, \theta_{r+1}$, aus, von denen wie üblich $\theta_{1}, \ldots, \theta_{r_{1}}$ den Körper $k$ im reellen $k^{\theta_{i}}$ und $\theta_{r_{1}+1}, \ldots, \theta_{r+1}$ im (untereinander nicht komplex-konjugierten) komplexen $k^{\theta_{i}}$ verwandeln. Wir setzen dann

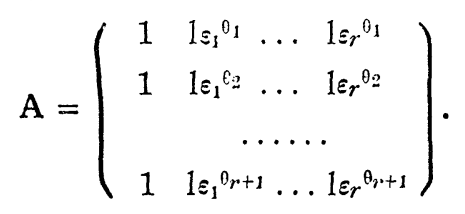

Addiert man in A jede Zeile zur letzten, so ergibt sich wegen $N \varepsilon= \pm 1$

$$
R(k)= \pm \frac{|\mathrm{A}|}{r+1} \text {. }
$$

Nun wollen wir den Regulator $R\left(\varepsilon H_{j}\right)$ der Gruppe $\varepsilon H_{j}$ betrachten. Dazu setzen wir wieder

$$
\mathrm{B}_{j}=\mathrm{B}\left(H_{j, 1}, \ldots, H_{j, s}\right)=\left(\begin{array}{cccc}
1 H_{j, 1}^{\theta_{1}} & \ldots & 1 H_{j, s}^{\theta_{1}} \\
1 H_{j, 1}^{\theta_{2}} & \ldots & 1 H_{j, s}^{\theta_{2}} \\
\ldots & \ldots & \ldots \\
1 H_{j, 1}^{\theta r+1} & \ldots & 1 H_{j, s}^{\theta r+1}
\end{array}\right)
$$

und schreiben der Kürze halber für ein Element $\sigma$ von $G$ 


$$
\sigma \mathrm{B}_{j}=\mathrm{B}\left(H_{j, 1}^{o}, \ldots, H_{j, s}^{o}\right)=\left(\begin{array}{ccc}
1 H_{j, 1}^{\sigma \theta_{1}} \ldots & \ldots H_{j, s}^{\sigma \theta_{1}} \\
\ldots & \ldots \\
1 H_{j, 1}^{\sigma \theta r+1} \ldots & 1 H_{j, s}^{\sigma \theta_{r+1}}
\end{array}\right)
$$

Es sei $\tau_{j}$ ein Element von $G$, das die Galoisgruppe von $\Omega_{j} / k$ erzeugt. Dann ist, wie bei (28),

$(29)(r+1) l R\left(\varepsilon H_{j}\right)=\left|\begin{array}{cc}\mathrm{A} & \mathrm{B}_{j} \\ \tau_{j} \mathrm{~A} & \tau_{j} \mathrm{~B}_{j} \\ \ldots \ldots & \ldots \\ \tau_{j}^{l-1} \mathrm{~A} & \tau_{j}^{l-1} \mathrm{~B}_{j}\end{array}\right|=\left|\begin{array}{cc}\mathrm{A} & \mathrm{B}_{j} \\ \mathrm{~A} & \tau_{j} \mathrm{~B}_{j} \\ \ldots \ldots \\ \mathrm{A} & \tau_{j}^{l-1} \mathrm{~B}_{j}\end{array}\right|=|\mathrm{A}| \cdot\left|\begin{array}{cc}\mathrm{E} & \mathrm{B}_{j} \\ \mathrm{E} & \tau_{j} \mathrm{~B}_{j} \\ \ldots \ldots . \\ \mathrm{E} & \tau_{j}^{l-1} \mathrm{~B}_{j}\end{array}\right|$,

wobei $\mathrm{E}^{\prime} \operatorname{die}(r+1)$-reihige Einheitsmatrix ist. Wird

$$
\Delta_{j}=\left|\begin{array}{cc}
\mathrm{E} & \mathrm{B}_{j} \\
\mathrm{E} & \tau_{j} \mathrm{~B}_{j} \\
\cdots \cdots \\
\mathrm{E} & \tau_{j}^{l-j} \mathrm{~B}_{j}
\end{array}\right|
$$

gesetzt, so ergibt sich nach (27), (29) und (30)

$$
\prod_{j=1}^{t} \frac{R\left(\varepsilon H_{j}\right)}{R(k)}=\frac{1}{l^{t}}\left|\prod_{j=1}^{t} \Delta_{j}\right| \text {. }
$$

8. Für die Grnppe $\varepsilon H_{\Omega}$ in $K$ ergibt sich ähnlich wie in 7

$$
\frac{R\left(\varepsilon H_{\Omega}\right)}{R(k)}=\frac{1}{l^{m}}|\Delta|
$$

wobei

$$
\Delta=\left|\begin{array}{cccc}
\mathrm{E} & \mathrm{B}_{1} & \ldots & \mathrm{B}_{t} \\
\mathrm{E} & \sigma_{2} \mathrm{~B}_{1} & \ldots & \sigma_{2} \mathrm{~B}_{t} \\
& \ldots & \ldots \\
\mathrm{E} & \sigma_{n} \mathrm{~B}_{1} & \ldots & \sigma_{n} \mathrm{~B}_{t}
\end{array}\right|
$$

ist. Ferner sei $\chi_{1}=1, \chi_{s}, \ldots, \chi_{n}$ die $n=l^{m}$ Charaktere von $G$ und sei

$$
X=\left|\begin{array}{ccc}
\mathrm{E} & \mathrm{E} \ldots & \mathrm{E} \\
\mathrm{E} & \chi_{2}\left(\sigma_{2}\right) \mathrm{E} \ldots \chi_{2}\left(\sigma_{n}\right) \mathrm{E} \\
& \ldots \ldots \chi_{n}\left(\sigma_{n}\right) \mathrm{E}
\end{array}\right|,
$$

so ist

$$
X \Delta=\left|\begin{array}{c}
\mathrm{A}_{1,0} \ldots \mathrm{A}_{1, t} \\
\ldots \ldots \\
\mathrm{A}_{n, 0} \ldots . \mathrm{A}_{n, t}
\end{array}\right|,
$$

wobei $\left(\sigma \mathrm{B}_{0}=\mathrm{E}\right)$

$$
\mathrm{A}_{i j}=\sum_{\sigma \in G} \chi_{i}(\sigma) \mathrm{E} \cdot \sigma \mathrm{B}_{j},(1 \leqq i \leqq n, 0 \leqq j \leqq t),
$$

sind. Aus der Charakterenrelation ersieht man leicht, dass $\mathrm{A}_{1,0}=l^{m} \mathrm{E}, \mathrm{A}_{9,0}=\ldots$ 
$=\mathrm{A}_{n, 0}=0$ sind. Für $i=1$ kö.nnen wir beweisen $\mathrm{A}_{1,1}=\ldots=\mathrm{A}_{\mathrm{1}, t}=0$, wie folgt. Es sei $U$ die Untergruppe von $G$, die dem $\Omega_{j}$ entspricht und sei $G=U+U_{\tau}+\ldots$ $+U \tau^{l-1}$. Weil dann $\tau=\tau_{j}$ eine erzeugende Substitution der Galoisgruppe von $\Omega_{j} / k$ ist, so ist nach (33) für $i=1,1 \leqq j \leqq t$

$$
\begin{gathered}
\mathrm{A}_{1, j}=\sum_{o \in G} \sigma \mathrm{B}_{j}=l^{m-1} \sum_{i=0}^{l-1} \tau^{a} \mathrm{~B}_{j}, \\
\sum_{a=0}^{l-1} \tau^{a} \mathrm{~B}_{j}=\sum_{a=0}^{l-1} \mathrm{~B}\left(H_{j, 1}^{\tau a}, \ldots, H_{j, s}^{\tau a}\right)=\mathrm{B}\left(N_{\Omega_{j} / k} H_{j, 1}, \ldots, N_{\Omega_{j} / k} H_{j, s}\right) .
\end{gathered}
$$

Da aber $N_{\Omega_{j} / k} H_{j, i}=1$ ist, so ist $\mathrm{A}_{1, j}=0$.

Um $\mathrm{A}_{i, j}$ für $i \neq 1, j \neq 0$ zu bestimmen, sei $V$ die Gruppe der Elemente $\sigma$ von $G$, für die $\chi_{i}(\sigma)=1$ sind. Falls $U=V$, so ist nach $(33)\left(\tau=\tau_{j}\right)$

$$
\mathrm{A}_{i j}=l^{m-} \sum_{a=0}^{l-1} \chi_{i}\left(\tau_{j}{ }^{a}\right) \mathrm{E}_{j}{ }^{a} \mathrm{~B}_{j}, \quad(i=2, \ldots, n ; j=1, \ldots, t) \text {. }
$$

Falls $U \neq V$, so sei $U \frown V=W, V=W+W \eta+\ldots+W \eta^{l-1}$, wo $\eta$ wieler eine die Gruppe von $\Omega_{j} / k$ erzeugende Substitution ist. Nach (33) ist in diesem Falle

Für festes $\sigma$ ist dann, wie oben,

$$
\mathrm{A}_{i, j}=\underset{\sigma \text { mod, } V}{l m-2} \mathcal{i}(\sigma) \mathrm{E} \cdot \sum_{a=1}^{l-1} \pi \eta^{a} \mathrm{~B}_{j}
$$

$$
\sum_{i=0}^{1-1} \sigma \eta^{a} \mathrm{~B}_{j}=\sigma \mathrm{B}\left(N_{\Omega_{j} / k} H_{j, \mathrm{l}}, \ldots, N_{\Omega_{j} / k} H_{j, s}\right)=0
$$

Daher sind im Falle $U \neq V$ alle $\mathrm{A}_{i, j}=0(i \neq 1, j \neq 0)$.

Für jedes $\Omega_{j}$ gibt es genau $l-1$ Charaktere $\%$, für welche die Gruppe von $\sigma$ mit $\chi(\sigma)=1$ mit der dem Körper $\Omega_{j}$ entsprechenden Untergruppe von $G$ übereinstimmt. Diese Charaktere werden mit $\chi_{1}^{(j)}, \ldots, \chi_{l-1}^{(j)}$ bezeichnet. In diesem Sinne lassen sich die $l^{m}-1$ Charaktere in $t=\left(l^{m}-1\right) /(l-1)$ Klassen von je $l-1$ Charakteren einteilen. Wir verabreden uns, dass die Charaktere $\chi_{n}, \ldots, \ell_{n}$ in der Determinante $X$ von vornherein so angeordnet werden, dass die Charaktere $\chi_{1}^{(j)}, \ldots, \chi_{i-1}^{j,}$ in der $j$-ten $l-1$ Reihen nebeneinandersteht. Dann ist

$$
X \Delta=\left|\begin{array}{cccc}
l^{m} \mathrm{E} & 0 & \ldots & 0 \\
0 & l^{m-1} \Gamma_{1} \ldots & 0 \\
& \ldots & \ldots & \\
0 & 0 \ldots & l^{m-1} \Gamma_{t}
\end{array}\right|,
$$

wobei $\mathrm{E}$ die $(r+1)$-reihige Einheitsmatrix und $\Gamma_{j}$ eine $s=(l-1)(r+1)$ reihige quadratische Matrix ist, nämlich wegen (34)

Daher erhalten wir

$$
\Gamma_{j}=\left(\begin{array}{c}
\sum_{a=0}^{l-1} \chi_{1}^{(j)}\left(\tau_{j}^{a}\right) \mathrm{E} \cdot \tau_{j}^{a} \mathrm{~B}_{j} \\
\cdots \cdots \\
\sum_{a=0}^{-1} \chi_{l-1}^{(j)}\left(\tau_{j}^{a}\right) \mathrm{E} \cdot \tau_{j}^{a} \mathrm{~B}_{j}
\end{array}\right) .
$$

$$
X \Delta=l^{m(r+1)+(m-1)(l-1)(r+1) t} \prod_{j=1}^{t}\left|\Gamma_{j}\right|
$$


9. Sei Ferner

$$
X_{j}=\left|\begin{array}{cccc}
\mathrm{E} & \mathrm{E} & \ldots & \mathrm{E} \\
\mathrm{E} & \chi_{1}^{(j)}\left(\tau_{j}\right) \mathrm{E} \ldots & \ldots \chi_{1}^{(j)}\left(\tau_{j}^{l-1}\right) \mathrm{E} \\
& \ldots \ldots & \ldots \\
\mathrm{E} & \chi_{l-1}^{(j)}\left(\tau_{j}\right) \mathrm{E} \ldots & \chi_{l-1}^{(j)}\left(\tau_{j}^{l-1}\right) \mathrm{E}
\end{array}\right|,
$$

so ist, wie oben nach (30)

$$
X_{j} \Delta_{j}=\left|\begin{array}{cc}
l \mathrm{E} & 0 \\
0 & \Gamma_{j}
\end{array}\right|=l^{r+1}\left|\Gamma_{j}\right| \quad(j=1, \ldots, t) .
$$

Wegen (22), (31), (32), (35) und (36) ist nun

$$
\mathbf{P}_{2}=l^{m-t}\left|\frac{\Pi \Delta_{j}}{\Delta}\right|=l^{m-t+(r+1)(t-m-(l-1)(m-1) t)}\left|\frac{X}{\Pi X_{j}}\right| \text {. }
$$

Da aber wegen der Orthogonalität der Charaktere

$$
\left|X^{2}\right|=l^{m(r+1) l^{m}}, \quad\left|X_{j}^{2}\right|=l^{(r+1) l}
$$

ist, so ist

$$
\mathbf{P}_{2}=l^{r(t-m)-\frac{r+1}{2}\left((m-1)\left(l^{m}-1\right)+t-m\right)} .
$$

Wegen (26) und (37) erhalten wir also den Ausdruck

$\mathbf{A}=\frac{\left(E: E_{\Omega}\right)}{l^{Q}}$,

(38) $Q=\frac{l^{u}-1}{l-1}-u+\frac{r+1}{2}\left((m-1)\left(l^{m}-1\right)+\frac{l^{m}-1}{l-1}-m\right)-r\left(\frac{l^{m}-1}{l-1}-m\right)$.

10. Die Zahl $\left(E: E_{\Omega}\right)$ ist ein Teiler von $l^{(m-1)(R+1)}$. Denn, da

$$
\prod_{j=1}^{t} N_{K / \Omega_{j}} E=E^{t-\left(l^{m-1}-1\right) /(l-1)}\left(N_{K / k} E\right)^{\left(l^{m-1}-1\right) /(l-1)}=E^{l^{m-1} \varepsilon}
$$

ist, so ist die $l^{m-1}$-te Potenz jeder Einheit von $K$ eine $E_{\Omega}$. Also folgt aus $\left(E: E^{l^{m-1}}\right)=\left(E: E_{\Omega}\right)\left(E_{\Omega}: E^{l^{m-1}}\right)$, dass der Index $\left(E: E_{\Omega}\right)$ ein Teiler von $\left(E: E^{l^{m-1}}\right)$ ist, die ihrerseits sicher ein Teiler von $l^{(m-1)(R+1)}$ ist.

Mathematisches Institut, Universität zu Nagoya 